\title{
Philosophiques
}

\section{Genette, Gérard (1994), L'œuvre de l'art, immanence et transcendance, Coll. Poétiques. Paris : Seuil.}

\section{Marie Martel}

Volume 26, numéro 1, printemps 1999

URI : https://id.erudit.org/iderudit/004955ar

DOI : https://doi.org/10.7202/004955ar

Aller au sommaire du numéro

Éditeur(s)

Société de philosophie du Québec

ISSN

0316-2923 (imprimé)

1492-1391 (numérique)

Découvrir la revue

Citer ce compte rendu

Martel, M. (1999). Compte rendu de [Genette, Gérard (1994), L'œuvre de l'art, immanence et transcendance, Coll. Poétiques. Paris : Seuil.] Philosophiques,

26(1), 111-115. https://doi.org/10.7202/004955ar

Ce document est protégé par la loi sur le droit d'auteur. L'utilisation des services d'Érudit (y compris la reproduction) est assujettie à sa politique d'utilisation que vous pouvez consulter en ligne.

https://apropos.erudit.org/fr/usagers/politique-dutilisation/ 


\section{GenetTe, Gérard (1994), L'œuvre de l'art, immanence et transcendance.}

Coll. Poétiques. Paris : Seuil.

Où est située l'œuvre d'art ? Dans l'objet matériel, dans l'esprit de son créateur, dans celui de ses interprètes (celui du profane ou de l'expert ?), ou ailleurs...? À cette question du statut ontologique de l'œuvre d'art, la tradition récente de l'esthétique analytique offre une nouvelle réponse. Une nouveauté qui a lieu d'étonner puisque l'auteur, G. Genette, littérateur de métier et artisan bien connu de la pensée française, vient avec cette contribution brouiller les cartes tant méthodologiques que géographiques, en faisant le saut en philosophie de l'art et en engageant un dialogue avec certains des interlocuteurs les plus éminents de la communauté anglo-saxonne dans ce domaine, peu célébrés et rarement traduits en France. Mais c'est du nouveau qui sonne un peu tout de même comme un air connu puisque l'ontologie de l'œuvre d'art défendue par l'A. se rallie à un consensus assez bien établi autour de la distinction goodmanienne entre œuvres autographiques et allographiques. L'adhésion au pluralisme ontologique qui découle de ce parti pris est motivée par la volonté de contrecarrer les " excès monistes » qui posent l'identification exclusive des œuvres tantôt à des objets physiques (en excluant les œuvres littéraires ou musicales), tantôt à des objets idéaux (en introduisant par exemple une confusion entre la longévité de l'artiste et la durée des œuvres si ces dernières sont identifiées à une des idées de son créateur) (p. 19). La première partie de l'ouvrage est consacrée au concept d'immanence, terme générique qui désigne les diverses façons dont une œuvre peut immaner en un objet ; elle répond à la question : "En quoi consiste l'œuvre ? " Suivant le critère empirique goodmanien repris par l'A., la pertinence pour certaines œuvres de la notion d'authenticité introduit une division catégorielle au sein des diverses pratiques artistiques. Certaines œuvres sont susceptibles d'être contrefaites, on dit qu'elles existent en régime autographique, leur identification dépend alors de l'histoire de production, du fait d'avoir été produites par un certain artiste dans certaines circonstances. S'il s'agit d'une peinture, par exemple, elle n'existe qu'en une seule instance, son objet d'immanence est conçu comme matériel. Pour d'autres productions artistiques, un texte littéraire, une composition musicale, il ne fait pas sens de parler de contrefaçon ou d'authenticité ; la présence d'une règle de correction leur permet d'être reproduites en un nombre illimité d'exemplaires tous aussi valables les uns que les autres, ce qui les établit comme allographiques. L'objet d'immanence de ces œuvres est idéal.

Une des qualités remarquables de l'ouvrage vient précisément de l'exploration de ce pluralisme ontologique qui donne lieu à un enrichissement de la distinction autographique/allographique, en montrant bien que celle-ci « traverse les arts sans les commander » et nous confronte à la spécificité ontologique de diverses pratiques artistiques qui opèrent sous un même label. La photographie, par exemple, n'est pas un art autographique de part en part. Dans le cas du polaroïd, on parle de régime autographique à objet d'immanence unique puisqu'un objet « irremplaçable dans sa singularité physique » est créé. La production d'épreuves négatives situe la photo en régime autographique multiple à cause de la présence de deux phases dont la première 
donne lieu à un modèle susceptible d'être contrefait, le négatif, duquel sera issu, dans la seconde phase, un groupe d'objets, les impressions, qui devront posséder la bonne histoire de production, c'est-à-dire être tirées de l'épreuve originale. On pourrait ajouter que, dans le cas des reproductions photographiques numérisées, la pratique de copies considérées comme identiques se veut déjà suffisamment établie pour déplacer la photographie en régime allographique. À une époque où les différences catégorielles entre les arts subissent des changements profonds et où naissent de nouvelles formes artistiques, l'A. rend justice à la fois à cette diversité et à la sensibilité de l'époque qui détermine historiquement certains de nos engagements ontologiques. Mais alors que l'on se serait attendu à ce que le pluralisme ressorte de cet exercice d'enrichissement plus renforcé que jamais, on peut se demander si cette observation minutieuse des objets en régime autographique n'a pas paradoxalement pour effet d'affaiblir cette thèse. À travers l'explicitation des décisions qui président dans chaque cas à la détermination de l'authenticité et du nombre des épreuves, il apparaît que seuls le dessin et la peinture sont véritablement des cas où l'incapacité matérielle à la reproduction est la cause de l'unicité des exemplaires. Selon l'A., dans la plupart des pratiques, les limitations sont volontaires, c'est-à-dire conventionnelles. Sans l'avoir cherché, cette exploration fait ressortir que le cas paradigmatique des objets en régime autographique unique incarné par le dessin, et même pas toujours par la peinture, fait figure d'exception dans la famille des arts et viole le principe d'unité théorique que l'on pourrait légitimement être tenté de revendiquer. Si l'on reconnaît que seules des limites techniques nous empêchent de défendre une thèse posant la possibilité de principe pour tous les arts d'avoir une multiplicité d'exemplaires, on ouvre la voie à une thèse moniste selon laquelle toutes les œuvres seraient des objets autographiques multiples ou allographiques (moyennant sans doute l'assouplissement de certaines contraintes dans ce dernier cas), ou encore ni un ni l'autre, mais un produit type ou un processus type à redéfinir.

La contribution la plus intéressante de l'ouvrage, et celle où l'A. se démarque le plus radicalement de la position de Goodman, réside dans son analyse des objets allographiques. L'A. défend une position aux antipodes métaphysiques du nominalisme goodmanien en décrétant plus efficace d'adopter une forme de structuralisme conceptuel posant l'existence idéale d'un objet d'immanence qui décrit exhaustivement l'ensemble des propriétés constitutives des œuvres littéraires ou musicales (p. 115). S'ils sont nombreux à concevoir l'identification d'un objet littéraire avec une structure type, peu se sont risqués à approfondir la nature de cette entité. Nominaliste ou conceptualiste, le point de départ est cependant le même : l'existence d'une œuvre en régime allographique tient à la possibilité de distinguer entre propriétés constitutives et contingentes, et l'itérabilité des premières rend l'œuvre indépendante de son histoire de production, condition nécessaire de l'allographisme. L'A. soutient quant à lui que l'exercice du régime allographique consiste en une opération mentale d'analyse des deux séries de propriétés, dont les propriétés contingentes, la grosseur et le type de caractère, la couleur de l'encre sont dites « propriétés de manifestation » alors que les propriétés sélectionnées comme constitutives, qui sont celles de l'identité orthographique, du texte, sont abstraites et constituent les " propriétés d'immanence ». L'auteur choisit de désigner l'opération instituant l'objet idéal d'immanence par le terme d'inspiration husserlienne "réduction allographique " plutôt que par " abstraction ", en soulignant que ces textes et ces mélodies que l'on rencontre au terme de cet exercice mental se distinguent en ceci du triangle qu'ils sont "idéaux sans être abstraits » (p. 103). Mais à quelle sorte d'idéalité a-t-on affaire ? Toujours en enrôlant 
la doctrine husserlienne, l'A. suggère que les textes ne sont pas des universaux comme les concepts, qui sont "libres et intemporels ", mais que ce sont des idéalités " enchaînées ", soumises à l'histoire par un acte de naissance ; et ce ne sont pas non plus des entités génériques mais bien des individus idéaux. Ainsi, le concept de poème est une classe dont un membre est "La mort des amants ", idéalité singulière, ou type, dont une occurrence, un token, cette copie de "La mort des amants » n'est pas elle-même un membre. L'idéalité de « La mort des amants » est bien celle d'un individu idéal dans la mesure où il ne possède pas de membre qui lui appartienne logiquement, il est ultime, il admet des prédicats comme " $\mathrm{x}$ est un poème " mais n'est prédiqué de rien puisqu'en effet on ne peut dire " ceci est une mort des amants » en référant à l'exemplaire de ce poème, dans la mesure un exemplaire de poème n'est pas un poème (p. 125-6)). Le caractère objectif, ou communicable, de cet individu idéal pourrait constituer un problème pour ce point de vue, comme c'est le cas dans bon nombre des thèses idéalistes, et l'A. n'ignore pas cet enjeu. Il propose, par le biais de l'exemple de Mozart qui entend le Miserere d'Allegri dans la chapelle Sixtine et qui le note en sortant, que les propriétés constitutives de l'œuvre " transférables ou convertibles de l'exécution à la partition mozartienne, sont bien en ce sens transcendantes à l'esprit et au cerveau de Mozart, et en ce sens objectives " (p. 117), sans développer davantage son propos. Du coup, on ne sait pas trop, faute d'un exposé plus généreux, si c'est bien dans le langage, ici la notation musicale, comme semble le suggérer l'exemple, que l'A. entrevoit une solution au problème de l'objectivité.

Dans la seconde partie, l'A. introduit un deuxième mode d'existence, la transcendance, dans le but de prendre en compte, dans la construction de l'identité des œuvres, les aspects qui sont complémentaires à la relation d'immanence à des objets matériels ou idéaux. Il y a transcendance par pluralité d'immanence en parlant d'une œuvre entendue comme existant en plusieurs objets « non tenus pour identiques et interchangeables " et auxquels la coutume attribue une " unité opérale » (p. 187) : les différentes traductions de Guerre et paix, les trois versions de La Tentation de StAntoine de Flaubert, les œuvres à répliques, reproduites par le procédé de l'autocopie pour des raisons souvent, mais pas toujours, commerciales, comme Chardin qui ne s'en est pas privé, avec quatre fois le Bénédicité. En outre, lorsqu'une œuvre se présente de manière lacunaire ou fragmentaire, comme la Vénus de Milo, ou lorsqu'on y accède par des voies indirectes au moyen de reproductions photographiques, de copies, il sera question de transcendance par partialité (p. 239). Enfin, un troisième mode, dit par pluralité opérale, veut rendre compte des cas où un même objet d'immanence est susceptible de revêtir des significations différentes en fonction des contextes de génération et, ce faisant, de supporter plusieurs œuvres (p. 259). On aborde ici une question chargée de controverses qui est au vortex des querelles sur le statut ontologique de l'œuvre d'art. L'enjeu est de déterminer si l'on doit inclure ou non les aspects de la provenance dans l'identité de l'œuvre : est-ce qu'une structure, un texte comme le Don Quichotte, si l'on reprend l'exemple canonique tiré de la fable de Borgès, peut être produit une fois par Cervantès et une autre fois par un auteur nommé Pierre Ménard, et donc être associé à plusieurs œuvres en fonction de différents contextes de génération ? Prenant encore une fois parti contre Goodman sur cette question, la thèse de l'oeuvre-comme-immanence-et-transcendance est favorable à la prise en compte des propriétés intentionnelles-historiques dans l'identité d'une œuvre ainsi qu'à l'idée d'une pluralité d'œuvres pour un même texte.

Mais cette thèse est-elle vraiment différente de celle de Goodman ? Rappelons que les propriétés constitutives de l'œuvre relèvent de l'objet d'immanence, mais ce ne sont pas là toutes les propriétés de l'œuvre, qui comprend aussi les propriétés qui 
relèvent de la transcendance. La transcendance se distingue de l'immanence comme ceci : elle « recouvre toutes les manières [...] dont une œuvre peut brouiller ou déborder la relation qu'elle entretient avec l'objet matériel ou idéal en lequel fondamentalement elle « consiste » [...]. En ce sens, la transcendance est un mode dérivé, un complément, parfois un supplément palliatif à l'immanence. Si l'on peut concevoir une immanence sans transcendance (...), on ne peut concevoir la transcendance sans immanence » (p. 185). Cette proposition ontologique peut être abordée de la même manière dont Gregory Currie (An Ontology of Art, 1989) a qualifié le structuralisme goodmanien, lequel admet la pertinence de la connaissance de l'histoire de production d'une œuvre pour l'exercice critique et appréciatif, mais sans faire reposer l'identité des œuvres sur ces aspects. Dans chacun des mondes possibles, l'œuvre est identifiée avec sa structure, qui correspond pour l'A. à l'objet d'immanence. Par ailleurs, au sein de ces différents mondes, les œuvres auront des histoires de production qui vont varier en fonction des sortes d'actions liées à la création et au contexte de production. On distingue entre les propriétés que l'œuvre possède essentiellement, c'est-à-dire celles qui sont possédées dans tous les mondes possibles, et celles qui sont non-essentielles, celles qui sont possédées dans certains mondes et pas dans d'autres. Dans cette perspective, l'objet d'immanence est considéré comme essentiel alors que les sortes d'actions qui constituent son histoire de production sont non-essentielles; par conséquent, les propriétés intentionnelles-historiques de transcendance résultant de celles-ci sont également traitées comme non-essentielles. La thèse de l'oeuvre-comme-immanence-ettranscendance admet bien que certaines des propriétés de l'œuvre relèvent de son contexte génétique, et l'A. dépense beaucoup d'énergie à défendre cette idée, mais il n'en demeure pas moins que, comme en ce qui concerne la thèse de l'oeuvre-en-tantque-texte de Goodman qui lui sert de repoussoir, les conséquences ontologiques sont tout aussi modestes puisqu'elle fait de l'objet d'immanence une composante essentielle alors que les propriétés de transcendance découlant de l'histoire de production sont vues comme non-essentielles. La proposition de l'A. nécessiterait donc certains aménagements pour constituer une alternative réelle à la thèse goodmanienne de l'oeuvre-en-tant-que-texte.

Par ailleurs, si l'on reprend l'exemple invoqué par l'A. et tiré de la fable de Borgès qui tient les prédicats « être archaïque » et « être pragmatique " pour vrais du texte de Ménard dans la perspective du $\mathrm{XX}^{\mathrm{e}}$ siècle alors que les prédicats incompatibles sont tenus pour vrais du texte de Cervantès dans la perspective du XVII ${ }^{\mathrm{e}}$ siècle, la thèse de l'A apparaît très problématique. Étant donné la proposition d'identifier l'œuvre essentiellement à l'objet d'immanence, il semble qu'une même œuvre, une même structure, un même objet d'immanence, soient sujets à l'attribution de propriétés incompatibles entre elles dans un même monde dans la mesure où, comme c'est le cas de l'exemple proposé, elles dérivent de contextes génétiques différents. Comme une chose ne peut posséder et ne pas posséder une propriété donnée dans un même monde, le même objet d'immanence ne peut pas être à la fois " archaïque » et ne pas être " archaïque ». Il semble donc que nous ayons ici un contre-exemple au point de vue immanentiste de l'identité des œuvres.

Mais, mis à part ces réserves, l'ouvrage est important à plusieurs égards. Dans le monde francophone d'une part, parce qu'en prenant position au sein de ce débat riche et fécond qu'est celui de la nature catégorielle des œuvres et en faisant habilement le pont entre deux horizons méthodologiques, l'A. insuffle de l'oxygène dans un climat intellectuel qui laisse souvent penser que la philosophie de l'art est 
moribonde sur le continent européen. D'autre part, son importance est indéniable pour le développement même de la problématique ontologique à cause du cadre original qu'il déploie pour penser la nature de l'œuvre comme individu idéal. Nombreuses, en effet, sont les propositions aujourd'hui qui sont prêtes à soutenir une ontologie qui rassemble toutes les œuvres sous un produit type, une structure avec une histoire de production, mais rares sont les contributions qui sont allées au-delà d'une suggestion nominale pour rendre compte de la nature de cette entité. Et si d'aventure on s'est parfois risqué plus loin, on a vu l'identification des œuvres se faire avec des êtres aux contours étranges, qualifiés par certains de "creatures of metaphysical darkness » et à côté desquelles les propositions de l'auteur semblent ouvrir une avenue de recherche fort saine et stimulante ; là encore, dans le monde quelquefois ténébreux de l'anglo-esthétique, on peut dire qu'il apporte une bouffée d'air frais. Enfin, ce présent examen de la condition d'artefact est suivi dans un ouvrage subséquent d'une analyse de la fonction esthétique et s'inscrit donc dans une entreprise plus vaste qui permet à l'A. d'oser la définition d' "artefact à fonction esthétique » pour les œuvres d'art en faisant fi du scepticisme qui règne presque dogmatiquement à l'égard des projets essentialistes en théorie de l'art.

MARIE MARTEL

Département de Philosophie

Université McGill 